\title{
FISH as an effective diagnostic tool for the management of challenging melanocytic lesions
}

\author{
Mathew W Moore* and Robert Gasparini
}

\begin{abstract}
Background: The accuracy of melanoma diagnosis continues to challenge the pathology community, even today with sophisticated histopathologic techniques. Melanocytic lesions exhibit significant morphological heterogeneity. While the majority of biopsies can be classified as benign (nevus) or malignant (melanoma) using well-established histopathologic criteria, there exists a cohort for which the prediction of clinical behaviour and invasive or metastatic potential is difficult if not impossible to ascertain on the basis of morphological features alone. Multiple studies have shown that there is significant disagreement between pathologists and even expert dermatopathologists in the diagnosis of this subgroup of difficult melanocytic lesions.
\end{abstract}

Methods: A four probe FISH assay was utilized to analyse a cohort of 500 samples including 157 nevus, 176 dysplastic nevus and 167 melanoma specimens.

Results: Review of the lesions determined the assay identified genetic abnormalities in a total of $83.8 \%$ of melanomas, and $1.9 \%$ of nevus without atypia, while genetic abnormalities were identified in $6.3 \%, 6.7 \%$, and $10.3 \%$ of nevus identified with mild, moderate and severe atypia, respectively.

Conclusions: Based on this study, inheritable genetic damage/instability identified by FISH testing is a hallmark of a progressive malignant process, and a valuable diagnostic tool for the identification of high risk lesions.

\section{Background}

Malignant melanoma is the seventh most common cancer responsible for $1-2 \%$ of all cancer related deaths [1] with the fastest increase in incidence identified as 3-7\% per year in the United States. Cutaneous malignant melanoma is a neoplasm which arises from melanocytes; it may occur de novo, or from a pre-existing lesion such as a congenital, acquired, or atypical (dysplastic) nevus. Noncutaneous primary sites of melanocytes include mucosal epithelium, retinas, and leptomeninges [2]. Melanoma is difficult to treat with therapy and most commonly fatal if not surgically removed prior to metastatic progression [3]. Early and accurate diagnosis of melanoma is therefore widely regarded as the most important factor in predicting outcome from this disease[4].

Successful management of potential melanomas is technically challenging. The initial lesion is usually discovered during either a routine physician visit, or

\footnotetext{
* Correspondence: mmoore@neogenomics.com

* Correspondence: mmoore@neogenomics.com FL 33913, USA
}

(c) 2011 Moore and Gasparini; licensee BioMed Central Ltd. This is an Open Access article distributed under the terms of the Creative

dermatology check-up. The "ABCD" method of physical examination [5], Asymmetry, Border irregularity, Color variegation, and a Diameter of $6 \mathrm{~mm}$ or more, in conjunction with a noticeable change over time, is typically utilized to determine the need for an initial biopsy.

Histopathologic analysis remains the gold standard for the pathologic diagnosis of melanoma. Numerous criteria for the histopathologic diagnosis of melanoma have been described including asymmetry, poor circumscription, cytologic atypia, mitotic activity, and failure of maturation with descent [6]. Even with these criteria, a considerable number of cases demonstrate conflicting morphologic criteria, and several studies have shown a level of intra-observer discordance at between $2-17 \%$ $[6,7]$. As Shoo et al. (2010) demonstrated, based on $1,500,000$ biopsies, and a discordance of $14.3 \%$, this equates to $\sim 214,500$ discordant cases in the USA annually.

\section{The Genetics of Melanoma}

The melanocyte is a neural crest-derived cell that localizes in humans to several organs including the 
epidermis, eye, inner ear and leptomeninges [8]. In the skin, melanocytes synthesize and transfer melanin pigments to surrounding keratinocytes, leading to skin pigmentation and protection against solar exposure. The combination of a long life cycle, constant ultraviolet light exposure and other environmental stresses, and a high rate of cell division coupled to replicative senescence [9] categorize melanocytes as a high risk cell type for genetic mutation and selection. Progression from melanocyte through dysplasia, and melanoma has been shown to be an evolutionary process requiring multiple genetic events that subsequently affect several oncogene and tumor suppressor pathways, which ultimately result in increasing dysplasia, and progression to invasion, and metastatic potential [10]. This is consistent with current literature models in other cancer types outlining a progression from a normal cell type through dysplasia, and finally malignancy.

The characterization of melanocytic progression to melanoma presents specific challenges derived from the diverse phenotypic nature of the disease. While it is generally regarded that benign nevi contain few or no genetic alterations, and melanoma are known to possess frequent gross genetic alterations [7] there continues to be widespread discussion on the fundamental underlying biology of dysplastic nevi. Central to the argument is the contested proposition of the nature of melanocytic dysplasia; are a subset of dysplastic nevi premalignant lesions of melanoma, or are all dysplastic nevi fundamentally benign and genetically distinct from melanoma? Morphologically, and phenotypically, this is unclear as many studies have shown a wide disparity in diagnosis even amongst experts in challenging cases $[11,12]$. Current molecular studies provide convincing evidence defining the dysplastic nevi group as a heterogeneous population of normal chromosomal complement; defined point mutations, and less commonly, demonstrating a high level of chromosomal instability and imbalance [13-16]. In contrast to this heterogeneous nature, congenital nevi contain a normal chromosomal complement (although "initiating" mutations in BRAF and NRAS amongst others have been identified), while conversely, melanoma as a group exhibits a high degree of genetic instability with multiple gains, losses, and rearrangements documented [14].

Recently, Gerami et. al. (2009) [7] described a Fluorescent in situ hybridization (FISH) based assay for distinguishing this "High Risk" group of nevi, based on copy number alterations of 3 genomic loci.. These regions contain the known cancer oncogenes CCND1 (11q23), MYB (6q22-q23) and RREB1 (6p25). Gerami et. al. (2009) demonstrated that these 3 loci were found predominantly in melanomas $(87 \%)$, while rarely $(5 \%)$ in congenital nevi. Significantly, a small percentage of dysplastic nevi (5\%) were also shown to harbour this mutation. This study was able to identify a subset of atypical nevi which later progressed to melanoma, and through retrospective analysis, demonstrated that this group of nevi harboured one or more of the CCND1, P16 or RREB1 copy number alternations, effectively demonstrating that atypical nevi possessing these mutations have both invasive and metastatic potential.

Given the inherent difficulty in phenotypically distinguishing dysplastic nevi from melanoma, this paper aims to expand on the previous studies demonstrating that there exists a subset of melanocytic lesions for which a confident distinction of nevi from malignant melanoma cannot be made morphologically, but which can be resolved through FISH analysis.

\section{Methods}

\section{Sample Set}

The sample set for the validation study consisted of samples of known melanoma, atypical nevus and benign nevus (Table 1). All samples were obtained from three commercial reference laboratories, and had undergone a standard clinical diagnostic workup, including

Table 1 Sample Set: Composition by Diagnosis.

\begin{tabular}{|c|c|c|c|}
\hline & Total Cases & Positive By FISH & \% Positive \\
\hline \multicolumn{4}{|l|}{ Nevus } \\
\hline Congenital & 8 & 0 & $0.00 \%$ \\
\hline Compound & 37 & 0 & $0.00 \%$ \\
\hline Intradermal & 62 & 1 & $1.60 \%$ \\
\hline Junctional & 9 & 0 & $0.00 \%$ \\
\hline Mild Atypia & 32 & 2 & $6.30 \%$ \\
\hline \multirow[t]{2}{*}{ Blue } & 9 & 0 & $0.00 \%$ \\
\hline & & Percent Positive & $1.90 \%$ \\
\hline \multicolumn{4}{|l|}{ Nevus-Dysplastic } \\
\hline Nevus-Moderate Atypia & 60 & 4 & $6.70 \%$ \\
\hline Nevus-Severe Atypia & 29 & 3 & $10.30 \%$ \\
\hline Nevus-Scalp & 38 & 3 & $7.90 \%$ \\
\hline Nevus-Spitz & 49 & 6 & $12.20 \%$ \\
\hline Subtotal & 176 & Percent Positive & $9.10 \%$ \\
\hline \multicolumn{4}{|l|}{ Melanoma } \\
\hline Superficial Spreading & 71 & 57 & $80.30 \%$ \\
\hline Spindle cell/desmo-plastic & 3 & 2 & $66.70 \%$ \\
\hline Nodular & 45 & 42 & $93.30 \%$ \\
\hline In Situ & 8 & 6 & $75.00 \%$ \\
\hline Metastatic & 40 & 33 & $82.50 \%$ \\
\hline Subtotal & 167 & Percent Positive & $83.80 \%$ \\
\hline Total Cases & 500 & & \\
\hline
\end{tabular}


hematoxylin and eosin stain (H\&E), and where required, special stains. Study sites were asked to provide as contiguous set of samples as possible (from each of the 3 groups) to limit selection bias. Samples were removed of all protected health information, and either blocks or slides were shipped to the study site. All samples were received with the original clinical diagnosis, which was retained by the study coordinator. Samples were subsequently re-examined and the original diagnosis reviewed by an internal board-certified dermatopathologist. A small number of cases for which a conflicting diagnosis was obtained were excluded from the validation study. Following the internal pathology review, all samples received an internal reference number, and the cases were blinded to the FISH technologists.

\section{Study Design}

This study followed the standard criteria for clinical validation of an analytical assay to the standards defined by the appropriate accrediting agencies. The following metrics were analyzed.

\section{Specificity}

The assay specificity was determined from the benign nevus group. Receiver operator curve (ROC) analysis and plots of sensitivity versus specificity were used to independently document the relative power of each probe set at various FISH cutoffs. Final assay cutoffs were selected to maximize specificity over sensitivity.

\section{Sensitivity}

Sensitivity was calculated as the percentage of unambiguous melanoma cases defined as positive by the 4-probe FISH assay using the derived FISH scoring cutoffs determined from the sensitivity experiment.

\section{Atypical Positivity Rate}

The percentage of nevi with moderate or severe atypia that are scored positive using the FISH scoring cutoffs derived from the specificity experiment were determined.

\section{FISH Hybridization}

$5 \mu \mathrm{m}$ tissue sections were baked at $65^{\circ} \mathrm{C}$ for $20 \mathrm{~min}$ and deparaffinized through multiple xylene washes and dehydrated with ethanol. Specimen slides were air dried and subsequently passed through a series of pre-treatment washes on the VP2000 Processor (Abbott Molecular, Abbott Park, Illinois, U.S.A.): $1 \mathrm{X}$ SSC $\left(80^{\circ} \mathrm{C}, 35\right.$ $\mathrm{min})$, de-ionized (DI) water $(3 \mathrm{~min})$, protease solution (10\% Pepsin in $0.2 \mathrm{~N} \mathrm{HCL} ; 37^{\circ} \mathrm{C}$ for $34 \mathrm{~min}$ ), DI water (3 min), increasing ethanol series $(70 \%, 80 \%, 100 \%$ ethanol, 2 min each). The probe cocktail; MYB (6q23) in SpectrumGold; CCND1 (11q13) in SpectrumGreen; RREB1 (6p25) in SpectrumRed and D6Z1 (6
Centromere) in SpectrumAqua (Abbott Molecular, Abbott Park, Illinois, U.S.A.) was manually dispensed onto the specimen and covered with a cover glass. Denaturation was performed on the Thermobrite (Abbott Molecular, Abbott Park, Illinois, U.S.A) at $74^{\circ} \mathrm{C}$ for $8 \mathrm{~min}$ followed by the hybridization at $37^{\circ} \mathrm{C}$ for 12 16 hrs prior to coverslip removal. The post-hybridization wash procedure on the VP2000 consisted of $2 \mathrm{X}$ SSC with $3 \%$ Tween $\left(70^{\circ} \mathrm{C}, 2 \mathrm{~min}\right)$ and $0.5 \%$ SSC prior to application of DAPI counterstain and signal enumeration.

\section{Enumeration of FISH Signals}

Slides were analysed, digitally documented, and automated probe signal enumeration performed with a Metafer Slide Scanning System (MetaSystems, Waltham, MA). The algorithm of Gerami et. al. (2009) was used for scoring, with adjustments for cutoff values as described in the discussion. All areas for automated analysis were selected by a dermatopathologist. All analyses were performed blinded of the specimen's diagnoses. Tumor-bearing areas were localized using the DAPI filter at low magnification. The tumor area was then thoroughly inspected for the presence of nuclei harbouring abnormal copy numbers of any probe. Areas with the most significant copy number changes were selected for automated enumeration. Wherever possible, a minimum of 3 abnormal areas were selected and within each area 10 random nuclei were analyzed under high-power $(64 \times$ oil objective). Overlapped or poorly hybridized nuclei were excluded from analysis. Samples in which less than 20 nuclei could be evaluated were excluded from the study.

\section{Results}

Table 2 summarizes study cohort assay results.

\section{Discussion}

Presented is an analysis of 500 cases, comprised of 157 nevi, 167 dysplastic nevi, and 176 melanoma cases. The four-fluor FISH assay correctly identified $83.8 \%$ of melanomas, and $98.1 \%$ of all benign nevi.

The four-fluor FISH panel utilized in this study identified $83.8 \%$ of true melanomas. This is consistent with previous FISH and array comparative genomic hybridization studies, and supports the previous literature which has clearly determined that melanomas typically exhibit a grossly altered genetic complement. In terms of clinical utility, the four probe panel is an effective compromise between cost, technical complexity and assay sensitivity. A four probe panel represents the maximum number of FISH probes that can be co-hybridized to a single section. Increasing the number of probes would increase the sensitivity, but also increase the technical complexity of the assay and associated clinical cost. 
Table 2 Study Results.

\begin{tabular}{|c|c|c|c|}
\hline & Total Cases & Positive By FISH & $\%$ Positive \\
\hline \multicolumn{4}{|l|}{ Nevus } \\
\hline Congenital & 8 & 0 & $0.00 \%$ \\
\hline Compound & 37 & 0 & $0.00 \%$ \\
\hline Intradermal & 62 & 1 & $1.60 \%$ \\
\hline Junctional & 9 & 0 & $0.00 \%$ \\
\hline Mild Atypia & 32 & 2 & $6.30 \%$ \\
\hline \multirow[t]{2}{*}{ Blue } & 9 & 0 & $0.00 \%$ \\
\hline & & Percent Positive & $1.90 \%$ \\
\hline \multicolumn{4}{|l|}{ Nevus-Dysplastic } \\
\hline Nevus-Moderate Atypia & 60 & 4 & $6.70 \%$ \\
\hline Nevus-Severe Atypia & 29 & 3 & $10.30 \%$ \\
\hline Nevus-Scalp & 38 & 3 & $7.90 \%$ \\
\hline Nevus-Spitz & 49 & 6 & $12.20 \%$ \\
\hline Subtotal & 176 & Percent Positive & $9.10 \%$ \\
\hline \multicolumn{4}{|l|}{ Melanoma } \\
\hline Superficial Spreading & 71 & 57 & $80.30 \%$ \\
\hline Spindle cell/desmo-plastic & 3 & 2 & $66.70 \%$ \\
\hline Nodular & 45 & 42 & $93.30 \%$ \\
\hline In Situ & 8 & 6 & $75.00 \%$ \\
\hline Metastatic & 40 & 33 & $82.50 \%$ \\
\hline Subtotal & 167 & Percent Positive & $83.80 \%$ \\
\hline Total Cases & 500 & & \\
\hline
\end{tabular}

Significantly, the RREB1 probe, at $60 \%$ exhibited the highest sensitivity for melanoma, while the CCND1 probe and MYB probes exhibited significantly lower sensitivities at $38 \%$ and $37 \%$ respectively. The derived cutoff values for each probe are presented in Table 3. Briefly, a case was considered positive if greater than $16 \%$ of scored cells for the RREB1 loci contained more than 2 signals; or greater than $22 \%$ of CCND1contained more than 2 signals; or more than $53 \%$ of nuclei contain more 6 p25 than centromere 6 signals; or more than $42 \%$ of nuclei contain fewer $6 \mathrm{q} 23$ than centromere 6 signals. The cutoff values presented in this study were similar, although lower than the previously published values of (29\%, 38\%, 55\%, and $42 \%$ respectively) [7]. These

Table 3 Derived Cutoff values by Probe

\begin{tabular}{cc}
\hline Loci & Cutoff Value \\
\hline RREB1 $>2$ & $16 \%$ \\
\hline CCND1 $>2$ & $19 \%$ \\
\hline RREB1 $>$ cen6 & $53 \%$ \\
\hline MYB $<$ cen6 & $42 \%$ \\
\hline
\end{tabular}

differences are most likely due to variation in scoring technique, cell selection, and equipment (manual verse automated) between the two studies. Applying the cutoff values of Gerami et. al. (2009) to the dataset of this study resulted in a sensitivity and specificity of $65 \%$ and 98.7\% respectively (data not shown). These differences highlight the importance of performing site specific validations for sensitivity and specificity prior to clinical application.

Review of the benign lesions determined the assay identified genetic abnormalities in less than $1 \%$ of nevi without atypia (compound, junctional, congenital, and intradermal), while genetic abnormalities were identified in $6.3 \%, 6.7 \%$, and $10.3 \%$ of nevus identified with mild, moderate and severe atypia respectively. These results are suggestive of a correlation between the severity of dysplasia and an increased probability of genetic abnormality and progression to melanoma. As it is standard practice to fully excise all dysplastic nevi, there is no opportunity to follow this cohort to provide direct evidence of morphologic progression to melanoma. While there is insufficient evidence to confirm the absolute malignancy of a lesion based solely on FISH analysis, the authors agree with the previous study's conclusions that the FISH positive dysplastic nevi group is of a significantly higher risk of progression to melanoma.

The assay did not identify one or more of the genetic abnormalities in $16.2 \%$ of all confirmed melanocytic lesions. This result infers that while these regions may be important in pathogenesis in some melanoma, they are clearly not causal in all melanomas. Both FISH and comparative genomic hybridization studies have previously been unable to identify a single genetic marker, or set of markers present in all samples, suggesting that the phenotypic representation of melanoma is actually comprised of several (or more) genetic "families", each with a unique underlying set of down regulated tumor suppressors and activated oncogenes driving pathogenesis. Similar degrees of genetic heterogeneity has been observed and studied in other cancers, and molecular characterization is being utilized clinically as an adjunct to traditional phenotypic markers to assist in diagnosis and treatment of many cancers including invasive breast carcinoma [3], lung cancer [17,18], prostate cancer [19], sarcomas [20], and renal tumors [21]. In invasive carcinoma of the breast, molecular profiling is routinely utilized in conjunction with traditional pathology to sub classify the tumors into molecular classes (basal-like, luminalA, luminal B, ERRBB2/Her2+), with different prognosis and different responses to specific therapies [3].

\section{Conclusions}

These results are consistent to the current literature models for cancer progression, characterized by a 
defined initiating event, followed by a series of degenerative progressive genetic mutations, phenotypic de-differentiation, activation of tumor oncogenes, inhibition of tumor suppressor genes, until the cell reaches a state of de-differentiation where invasion and metastatic potential is possible. While the genetics behind this progression is well studied in several malignancies, there is still significant disagreement in the melanoma community as to the correlation (if any) between genetic instability and phenotypic dysplasia or atypia. While there continues to be significant discussion in pathology texts on the role of atypic presentation in the progression of melanocytic nevi to melanoma, genetically, it is clear that the process from melanocyte to melanoma requires multiple initiating events, followed by genomic instability, and finally progression to phenotypically evident malignancy.

Based on these results, the four-fluor FISH assay accurately identifies a molecular signature which is consistent with lesions that have invasive and metastatic potential. Lesions with these genetic qualities should be considered as possessing a genetic signature consistent with invasion and metastatic potential, even if this is not evident by morphology alone.

Clinically, the utility of this assay is predicated on the high specificity, and even in the absence of a conclusive melanoma diagnosis, the authors believe that a positive FISH case with corresponding atypical morphology should be treated as a high risk lesion and may warrant a wider excision, and more routine follow-up. Due to the moderate sensitivity of $83.8 \%$, care must be taken in the analysis of dysplastic nevi which are negative by FISH, with the inference being that almost $17 \%$ of melanomas are driven by a molecular profile which is undetected through this assay. Conversely, a negative FISH result may provide additional re-assurance to the pathologist in cases where a benign result is expected, and excision is difficult or undesired. When accompanied by morphological risk factors, a positive FISH result is a valuable diagnostic tool for the identification of high risk lesions.

\section{Acknowledgements}

Fumiko Yamamoto, Melinda Kwok, Dulce Amor Butoi, Hung Pham, Alfonso Del Campo and Michelle Marcial, assisted in performing the FISH hybridizations. Suman Verma and Philip Cotter contributed to the manuscript review and draft.

\section{Authors' contributions}

MWM and RG conceived of the study, and participated in its design and coordination. MWM supervised and carried out the studies. MWM wrote the manuscript, and RG contributed to the manuscript review and draft. All authors read and approved the final manuscript.

\section{Competing interests}

Robert Gasparini (RG) and Mathew Moore (MWM) are both employees of NeoGenomics, a company specializing in clinical oncology, including the diagnosis of melanocytic lesions. This study was performed in part to determine if FISH could be used at NeoGenomics to improve the accuracy of diagnosis of challenging melanocytic lesions.

Received: 19 June 2011 Accepted: 11 August 2011

Published: 11 August 2011

\section{References}

1. Jemal A, Siegel R, Ward E, Hao Y, Xu J, Murray T, Thun MJ: Cancer statistics, 2008. CA: a cancer journal for clinicians 2008, 58:71-96.

2. Newman MD, Lertsburapa T, Mirzabeigi M, Mafee M, Guitart J, Gerami P: Fluorescence in situ hybridization as a tool for microstaging in malignant melanoma. Modern pathology: an official journal of the United States and Canadian Academy of Pathology, Inc 2009, 22:989-995.

3. Geyer FC, Marchio C, Reis-Filho JS: The role of molecular analysis in breast cancer. Pathology 2009, 41:77-88.

4. Cassileth BR, Clark WH Jr, Lusk EJ, Frederick BE, Thompson CJ, Walsh WP: How well do physicians recognize melanoma and other problem lesions? Journal of the American Academy of Dermatology 1986, 14:555-560.

5. Friedman RJ, Rigel DS, Kopf AW: Early detection of malignant melanoma: the role of physician examination and self-examination of the skin. CA: a cancer journal for clinicians 1985, 35:130-151.

6. Shoo BA, Sagebiel RW, Kashani-Sabet M: Discordance in the histopathologic diagnosis of melanoma at a melanoma referral center. Journal of the American Academy of Dermatology 2010, 62:751-756.

7. Gerami P, Jewell SS, Morrison LE, Blondin B, Schulz J, Ruffalo T, Matushek Pt, Legator $M$, Jacobson K, Dalton SR, et al: Fluorescence in situ hybridization (FISH) as an ancillary diagnostic tool in the diagnosis of melanoma. The American journal of surgical pathology 2009, 33:1146-1156.

8. Bandyopadhyay D, Timchenko N, Suwa T, Hornsby PJ, Campisi J, Medrano EE: The human melanocyte: a model system to study the complexity of cellular aging and transformation in non-fibroblastic cells. Experimental gerontology 2001, 36:1265-1275.

9. Mooi WJ, Peeper DS: Oncogene-induced cell senescence-halting on the road to cancer. The New England journal of medicine 2006, 355:1037-1046.

10. Hanahan D, Weinberg RA: Hallmarks of cancer: the next generation. Cell 2011, 144:646-674

11. Farmer ER, Gonin R, Hanna MP: Discordance in the histopathologic diagnosis of melanoma and melanocytic nevi between expert pathologists. Human pathology 1996, 27:528-531.

12. Lodha S, Saggar S, Celebi JT, Silvers DN: Discordance in the histopathologic diagnosis of difficult melanocytic neoplasms in the clinical setting. Journal of cutaneous pathology 2008, 35:349-352.

13. Bastian $B C$, Olshen $A B$, LeBoit PE, Pinkel D: Classifying melanocytic tumors based on DNA copy number changes. The American journal of pathology 2003, 163:1765-1770.

14. Curtin JA, Fridlyand J, Kageshita T, Patel HN, Busam KJ, Kutzner H, Cho KH Aiba S, Brocker EB, LeBoit PE, et al: Distinct sets of genetic alterations in melanoma. The New England journal of medicine 2005, 353:2135-2147.

15. Moore SR, Persons DL, Sosman JA, Bobadilla D, Bedell V, Smith DD, Wolman SR, Tuthill RJ, Moon J, Sondak VK, Slovak ML: Detection of copy number alterations in metastatic melanoma by a DNA fluorescence in situ hybridization probe panel and array comparative genomic hybridization: a southwest oncology group study (S9431). Clinical cancer research: an official journal of the American Association for Cancer Research 2008, 14:2927-2935.

16. Morey AL, Murali R, McCarthy SW, Mann GJ, Scolyer RA: Diagnosis of cutaneous melanocytic tumours by four-colour fluorescence in situ hybridisation. Pathology 2009, 41:383-387

17. Boutros PC, Lau SK, Pintilie M, Liu N, Shepherd FA, Der SD, Tsao MS Penn LZ, Jurisica I: Prognostic gene signatures for non-small-cell lung cancer. Proceedings of the National Academy of Sciences of the United States of America 2009, 106:2824-2828.

18. Hayes DN, Monti S, Parmigiani G, Gilks CB, Naoki K, Bhattacharjee A Socinski MA, Perou C, Meyerson M: Gene expression profiling reveals reproducible human lung adenocarcinoma subtypes in multiple independent patient cohorts. Journal of clinical oncology: official journal of the American Society of Clinical Oncology 2006, 24:5079-5090.

19. Taylor BS, Schultz N, Hieronymus H, Gopalan A, Xiao Y, Carver BS, Arora VK, Kaushik P, Cerami E, Reva B, et al: Integrative genomic profiling of human prostate cancer. Cancer cell 2010, 18:11-22. 
20. Dang TA, Man TK: Classification of sarcomas using bioinformatics and molecular profiling. Current pharmaceutical biotechnology 2007, 8:83-91.

21. Youssef YM, White NM, Grigull J, Krizova A, Samy C, Mejia-Guerrero S,

Evans A, Yousef GM: Accurate molecular classification of kidney cancer subtypes using microRNA signature. European urology 2011, 59:721-730.

doi:10.1186/1746-1596-6-76

Cite this article as: Moore and Gasparini: FISH as an effective diagnostic tool for the management of challenging melanocytic lesions. Diagnostic Pathology 2011 6:76.

Submit your next manuscript to BioMed Central and take full advantage of:

- Convenient online submission

- Thorough peer review

- No space constraints or color figure charges

- Immediate publication on acceptance

- Inclusion in PubMed, CAS, Scopus and Google Scholar

- Research which is freely available for redistribution

Submit your manuscript at www.biomedcentral.com/submit
Ciomed Central 